Bulletin of Pharmaceutical Sciences
Assiut University
Website: http://bpsa.journals.ekb.eg/
e-mail: bullpharm@aun.edu.eg

\title{
ANTIBIOTIC RESISTANCE OF ACINETOBACTER BAUMANNII: AN URGENT NEED FOR NEW THERAPY AND INFECTION CONTROL
}

\author{
Randa Abd-ElNasser ${ }^{1,2^{*}}$, Mona Hussein Abdel-Rahim ${ }^{1}$, Manal Ahmed Mahmoud ${ }^{3}$, \\ Amal Ahmed Elkhawaga ${ }^{1}$ and Khaled Mohamed Hassnein ${ }^{1}$ \\ ${ }^{1}$ Department of Medical Microbiology and Immunology, Faculty of Medicine, Assiut \\ University, Assiut, Egypt \\ ${ }^{2}$ Ministry of Health and Population, Assiut, Egypt \\ ${ }^{3}$ Department of Chest Diseases, Faculty of Medicine, Assiut University, Assiut, Egypt
}

\begin{abstract}
Acinetobacter baumannii (A. baumannii) infections became an emerging health concern in hospitals across the globe and are often related to nosocomial infections with poorer clinical outcomes in patients with prolonged hospital stay. Management of infections involves prompt identification of the infecting strain, isolating the source of infection, and proper choice of antibiotic regimen. However, resistance to first-line antimicrobial drugs, combined with a scarcity of equally effective alternatives, complicates the treatment of multidrug-resistant (MDR) A. baumannii. Presently, MDR A. baumannii may be a serious health concern in hospitals and long-term care facilities accounting for up to $20 \%$ of infections in intensive care units, and $7 \%$ of infections in patients who are physically connected to medical equipments. Immediate and sustained prevention efforts are needed to control the speed of incidence. Antibiotic use is largely under-regulated in Egypt leading to the emergence of resistant isolates. This review describes genetic markers and other factors that influence the incidence of MDR A. baumannii. Current and emerging treatments as well as infection control strategies are discussed.
\end{abstract}

\section{INTRODUCTION}

Acinetobacter baumannii (A. baumannii) is a Gram-negative coccobacillus, non-motile, catalase-positive, oxidase-negative, nonfastidious, and strictly aerobic bacterium that has become a growing problem in hospitals as a predominant multidrug-resistant (MDR) bacterium in the intensive care and burn units. A. baumannii is considered an important source of nosocomial infections and is currently considered one of the pacesetters in the antibiotic resistance crisis ${ }^{1}$.

The evolution of MDR A. baumannii is well documented and is generally characterized by increasing resistance to the first- and second-generation cephalosporins in the 1970s followed by the reports of imipenem resistance in the 1980s and 1990s. Since then, several outbreaks have been reported in Asia, the Middle East, Europe, and North and South America $^{2-4}$. MDR A. baumannii infections are generally defined by resistance to three or more representatives from the quinolone, cephalosporin, $\beta$-lactam, aminoglycoside, and carbapenem families of antibiotics 5 .

According to the Centers for Disease Control and Prevention, the majority of clinically relevant strains of A. baumannii are MDR which accounts for up to $20 \%$ of infections in intensive care units, and for $7 \%$ of infections in patients who are physically connected to medical equipment ${ }^{6}$. Also in critically ill patients in intensive care units infected with MDR A. baumannii, there is a substantial rise in patient mortality rates ${ }^{7}$. In the USA, about 12,000 A. baumannii infections occur yearly with 500 deaths associated with

Received in 29/8/2020 \& Accepted in 12/10/2020 
these infections ${ }^{8}$. In Egypt $A$. baumannii infections grows rapidly in worrisome way, Many studies detected that $A$. baumannii infections may be associated with considerable mortality, however some of them support the possibility that the clinical course of debilitating patients may be influenced by many factors that subsequently the infection with $A$. baumannii may not independently lead to worst results ${ }^{9}$. The mortality from Acinetobacter infection was from $30 \%$ to $50 \%$ of Acinetobacter infected patients ${ }^{10}$. Sentinel site surveillance in tertiary care hospitals in Egypt showed high HAI rates ${ }^{11}$. During the last decade, while infection prevention and control (IPC) activities were progressing in Egypt, it was deemed important to implement a standardized national HAI surveillance program to define the magnitude and scope of HAIs in the country and to allow for interhospital comparisons of HAI rates. Therefore, a plan to implement a nationwide HAI surveillance program in intensive care units(ICUs) was developed with support from several partners: the U.S. Centers for Disease Control and Prevention's (CDC's) Global Disease Detection (GDD) Program in Egypt, the U.S. Naval Medical Research Unit (NAMRU-3), and the U.S. Agency for International Development in Egypt. The objectives of the national HAI surveillance were to estimate the incidence of HAIs, obtain national benchmarks, describe the microbiologic profile of pathogens causing HAIs, and inform prevention activities of HAIs. This report describes the process of developing a national HAI surveillance program in Egypt ${ }^{12}$. The aim of this review is to describe mechanisms of antibiotic resistance in A. baumannii, and focus on the emergency of spread of multi drug resistant $A$. baumannii and the need to current and emerging treatments as well as infection control strategies.

\section{Mechanisms of antibiotic resistance}

The capacity of A. baumannii to rapidly alter its genome and the ability to survive on inanimate surfaces and medical equipment with high resistance to disinfectants have established MDR A. baumannii as a frequent cause of hospital outbreaks ${ }^{13}$. The antibiotic resistance phenotype in A. baumannii is believed to be mediated by a combination of factors including upregulation of the organism's innate resistance mechanisms, lateral gene transfer, gene amplification, and gain/loss of function as a result of mutations ${ }^{14}$. Rapid acquisition of drug-resistant phenotype in A. baumannii is often associated with the presence of genomic regions referred to as 'resistance islands', which can be found on the bacterial chromosomes and/or plasmids in regions that are interspersed with mobile genetic elements ${ }^{15}$. AbaR1 is an $86 \mathrm{~kb}$ resistant island that is a product of repetitive insertion of various mobile genetic elements and is often associated with the dissemination by homologous recombination through lateral gene transfer with the genera Pseudomonas, Salmonella, and Escherichia ${ }^{16}$. In antibioticsusceptible A. baumannii, AbaRl is shown to correspond to a $20 \mathrm{~kb}$ ATPase-like open reading frame that acts as a specific hotspot of genomic instability, enabling the organism to change its genome in response to the environmental pressures ${ }^{16}$. Genomic islands such as AbaRl confer resistance to various antibiotics by carrying a cluster of genes encoding the proteins related to antibiotic inactivation. A. baumannii strains may become resistant to aminoglycosides by the acquisition of resistance islands that code for Aminoglycoside - Modifying Enzymes (AMEs) $)^{14}$. The genes encoding AMEs can be found on plasmids and/or transposons and include aacCl, aphA6, aadA1, and others that are grouped according to the type of enzyme activity ${ }^{14}$. A report by Namvar et al. examined the prevalence of AME genes in MDR A. baumannii isolates ${ }^{17}$. More than $98 \%$ of $A$. baumannii isolates had aacCl and aphA6 genes, whereas $80.8 \%$ of strains had all three aacC1, aphA6, and aadAl genes. The presence of aphA1, aacCl, or aphA6 was also found to confer resistance to kanamycin, gentamicin, or kanamycin and amikacin combined therapy ${ }^{18}$.

The OXA-type (oxacillin-hydrolysing) enzymes are among the earliest detected plasmid-mediated $\beta$-lactamases. Which usually confers resistance to penicillins and possesses high-level hydrolytic activity against oxacillin $^{19}$. A study by Davadeh et al. used 76 carbapenem-resistant $A$. baumannii isolates from a hospital in Turkey. Using polymerase chain reaction amplification methods, 22 strains were identified with OXA-51-like, 
OXA-23-like, OXA-40-like, and OXA-58-like genes $^{20}$. The higher carbapenem hydrolysis rate occurs due to the acquisition of the insertion sequence (IS) elements (e.g. ISAbal), which are from a naturally occurring plasmid upstream of the OXA-type carbapenemase encoding genes ${ }^{21}$. Others have reported that OXA-40 has replaced other oxacillinases such as OXA-23 as the main factor affording resistance to imipenem ${ }^{22}$. A. baumannii in clinical isolates often confers resistance to cephalosporins. The resistance profile is controlled by non-inducible $A m p C$ cephalosporinase that is controlled by an upstream ISAbal ${ }^{23}$. Mechanisms of carba-penem resistance are illustrated in figure $1^{24}$.

Boinett et al. have recently reported on MDR A. baumannii which is resistant to antibiotics that target lipid $\mathrm{A}^{25}$. The genes responsible for the modification include IpxA, Ip $x C$, and IpxD that encode the lipid A component of lipopolysaccharides (LPS). The mutations in these genes cause a loss of LPS, and since the drug action requires the binding of LPS, the drugs are rendered ineffective. A study by Moffatt et al. examined 13 derivatives of MDR A. baumannii strains. From the parent strain of A. baumannii (ATCC 19606), derivatives were generated with the mutations in Lipid A genes. There was a strong correlation between mutation in IpxA gene and the emergence of MDR A. baumannii ${ }^{26}$.
Moreover, AdeABC efflux system genes also a contributing factor in the emergence of resistance against certain antibiotics ${ }^{27}$. The efflux system is controlled by clustered genes ade $A$, adeB, and adeC, which were found to encode proteins for membrane fusion, drug transporter, and outer membrane component ${ }^{28}$. Expression of this system is regulated by two genes: a two-step regulator, $a d e R$, and a sensor, adeS. Peleg et al. reported that a point mutation in adeR and adeS may lead to reduced tigecycline susceptibility in A. baumannii due to the $A d e A B C$ pump overexpression ${ }^{13}$. A study by Nemec et al. examined $116 \mathrm{~A}$. baumannii strains for the presence of the structural genes $a d e A$, adeb, and ade $C$ and of the regulatory genes adeS and $a d e R$; at least one of the five genes of AdeABC complex was found in $91 \%$ of strains ${ }^{29}$. Efflux pumps also reduce the intracellular accumulation of other drugs such as fluoroquinolones and aminoglycosides.

However, other primary mechanisms, such as gyrase gene mutation for fluoroquinolones and AMEs acquisition for aminoglycosides, are often required to confer resistance phenotype to the corresponding antibiotics, suggesting a secondary rather than a primary role for AdeABC efflux system in the emergence of aminoglycoside- and fluoroquinolone-resistant A. baumannii strains ${ }^{14}$. Different mechanisms of antibiotic resistance of $A$. baumanni are revealed in figure $2^{24}$.

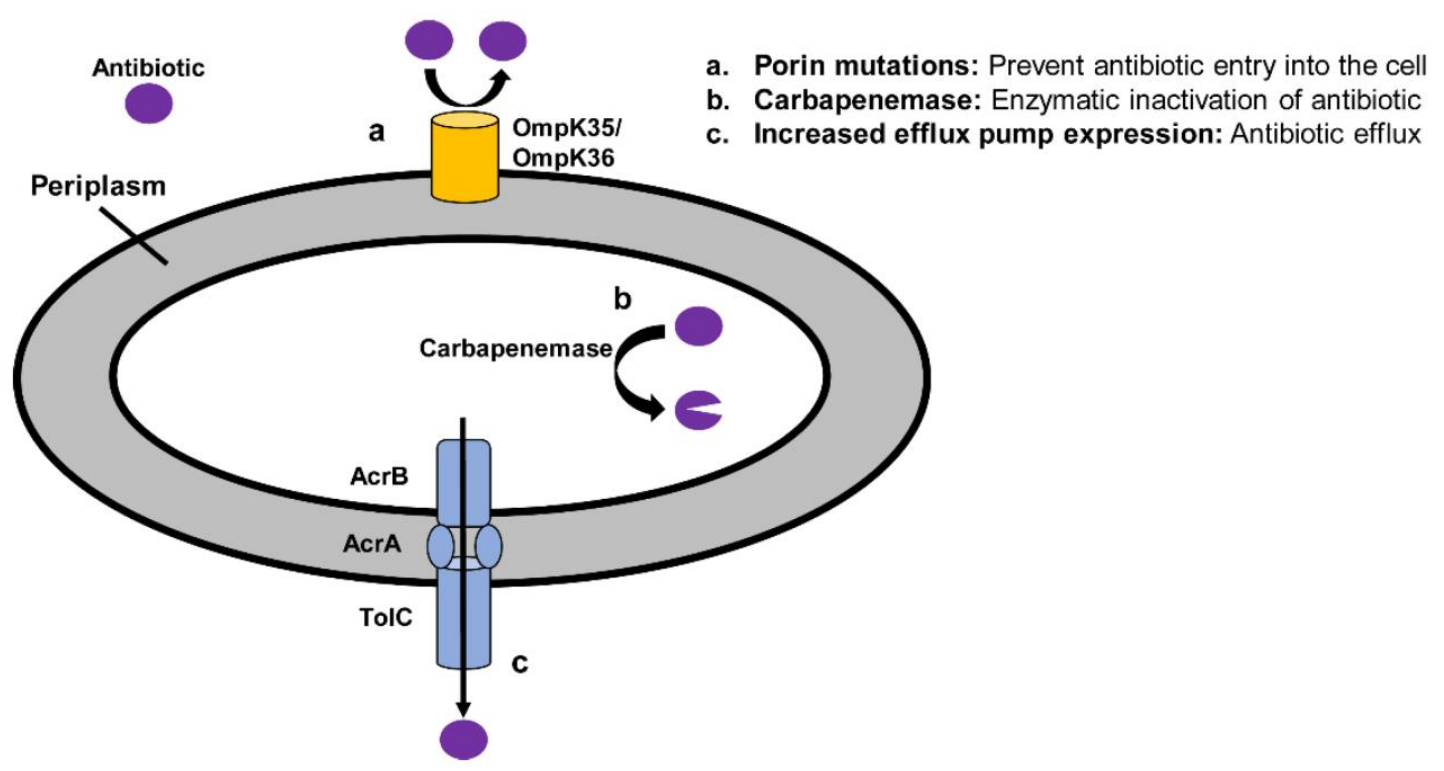

Fig. 1: Mechanisms of carbapenem resistance in A. baumannii. 


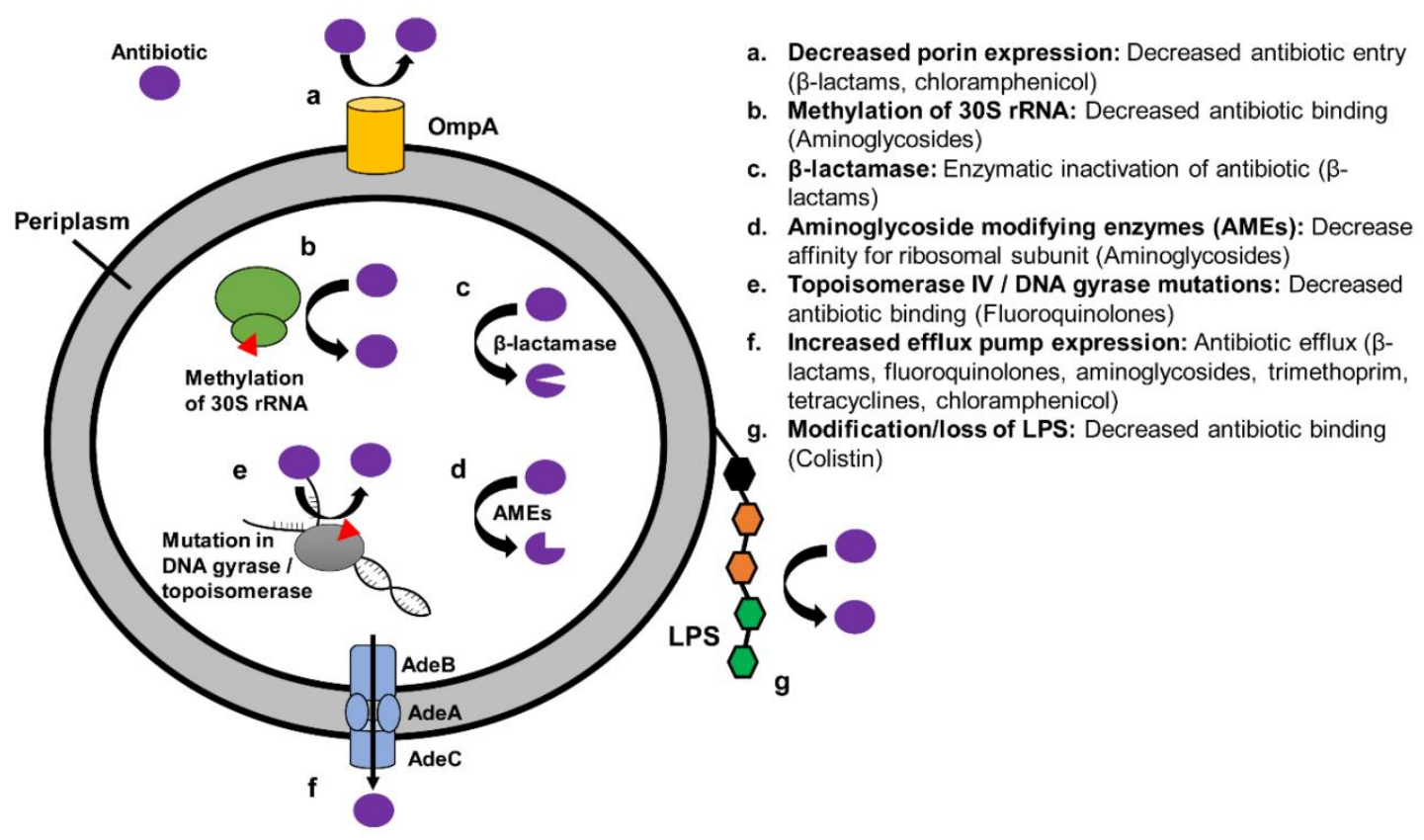

Fig. 2: Mechanisms of antibiotic resistance in A. baumannii.

\section{Treatment options for A. baumannii infections}

A. baumannii has the capacity to rapidly develop resistance through intrinsic and acquired mechanisms, thus the available treatments for $A$. baumannii infections are limited. Carbapenems used against Acinetobacter infections, but resistance rates have substantially risen in recent years.

Polymyxins show reliable antimicrobial activity against A. baumannii isolates. Polymyxin antibiotics are now considered to be the drug of choice for treating infections caused by carbapenem-resistant strains ${ }^{30}$.

However, polymyxin application is also a key driver for the emergence of adaptive polymyxin resistance with the potential of generating highly polymyxin-resistant bacterial populations ${ }^{31}$.

Tigecycline and its derivative have also shown high antimicrobial activity against $A$. baumannii. This class of antibiotics, however, is associated with higher in-hospital mortality and longer hospital stay ${ }^{32}$.

Sulbactam has also been successfully used in the treatment of serious A. baumannii infections $^{33}$. Sulbactam is relatively inexpensive and more efficient against $A$. baumannii when used as monotherapy than the ampicillin-sulbactam combination. Sulbactam alone is not available in many countries including the $\mathrm{USA}^{34}$. Therefore, a higher dosage of ampicillin-sulbactam combination regimen is needed for effective dosage against A. baumannii infections ${ }^{33}$.

Aminoglycosides are also an important group of antibiotics in treatment of aerobic Gram-negative bacteria including $A$. baumannii, but recent reports indicate the emergence of resistance to aminoglycosides in Acinetobacter isolates worldwide ${ }^{17}$.

Because of the decline in susceptibility to a wide range of antibiotic agents, the antibiotic options are often limited to synergistic application of these agents.

Based on in-vitro studies and observational data, the antibiotic options often used as monotherapy or in combination therapies to treat MDR A. baumannii infections are discussed below.

\section{Polymyxins (cell membrane inhibitors)}

Polymyxin antibiotics inhibit bacterial membranes after binding to lipopolysaccharides, interacting with lipid A of the outer membrane, and acting as a detergent by disrupting the membrane phospholipids ${ }^{35}$. Polymyxins are often used as the antibiotic therapy of choice against MDR A. baumannii ${ }^{35}$. 
Colistin, also known as polymyxin E, is administered intravenously as an inactive prodrug, which then converts into active colistin in blood. The process of converting prodrug to active colistin can take up to several hours to reach the desired concentration in the plasma. Therefore, this delayed initiation may be associated with the increased mortality of clinically ill patients, and the low colistin concentration may be linked to the emergence of a colistin-resistant population ${ }^{36 \& 37}$. Also the application of colistin may raise the probability of nephrotoxicity and neurotoxicity complications $^{38}$.

Polymyxin B is administered as an active antibiotic that rapidly achieves the desired plasma concentration and is highly effective against $A$. baumannii. Several studies ${ }^{39}$ have reported that polymixin $B$ is the most effective antibiotic for Acinetobacter strains. Zorgani et al., Attia and ElBaradei, Josheghani et al., currently, the use of polymyxin B combined with other antibiotic agents, such as carbapenems, rifampicin, and sulbactam, is recommended antibiotic therapy treatment for pneumonia and bacteraemia infections caused by MDR $A$. baumannii ${ }^{40-42}$.

Leite et al., reported that colistin resistance in A. baumannii isolates in Brazil reached $35 \%$. Inhaled colistin has also been recommended for treating pneumonia caused by $A$. baumannii ${ }^{44}$; however, not all studies support the benefits of inhaled colistin in patients with pneumonia since colistin yields a low concentration and a higher risk of bronchoconstriction $^{45}$.

\section{Tigecycline (ribosomal inhibition)}

Tigecycline is a derivative of minocycline that inhibits protein synthesis by binding to the 30 S ribosomal subunit and so blocking the interaction of aminoacyl-tRNA with the A site of the ribosome. Tigecycline is an alternative antibiotic choice for treating MDR $A$. baumannii. In the study by Kim et al. in patients with pneumonia caused by MDR $A$. baumannii, the clinical success rates of treatments were similar between those who received tigecycline-based and those who received colistin-based treatments ${ }^{46}$. Aljindan et $a l .{ }^{47}$, reported that tigecycline resistance of Acinetobacter isolates reached $17.72 \%$.

\section{Aminoglycosides (protein synthesis inhibition)}

Aminoglycosides are a broad-spectrum bactericidal agent that inhibits protein synthesis by binding to the $30 \mathrm{~S}$ subunit of bacterial ribosomes, resulting in the release of premature proteins by misreading the $\mathrm{mRNA}^{48}$. Unlike tigecycline, the result of aminoglycosides is a non-functional protein, and the efficacy of aminoglycosides in treating infections increases with increasing concentrations of the $\operatorname{drug}^{17}$. Aminoglycosides may be used in combination therapy with $\beta$-lactams ${ }^{49}$.

A. baumannii resistance to aminoglycosides is due to the mutations in the genes coded for aminoglycoside-modifying enzymes (AMEs). The mutated enzymes inactivate the drug and prevent ribosomal binding by catalyzing the modification at hydroxyl or amino groups of antibacterial agents. The degree of aminoglycoside resistance is influenced by multiple factors including the acquisition of enzyme-modifying genetic elements (lateral gene transfer), a decrease in the uptake of drugs in the cytoplasm (e.g. efflux pumps), and structural change in the $30 \mathrm{~S}$ ribosome (chromosomal mutations) ${ }^{49}$.

\section{Emerging therapies Iron chelation therapy}

Previous studies with iron chelators have demonstrated a bacteriostatic effect on bacterial growth $^{50}$. To survive and replicate, bacteria need iron for DNA replication and energy production; so iron chelation therapies have been exploited to target iron metabolism and achieve antibacterial activity by interrupting iron recruitment ${ }^{51}$.

To obtain a steady supply of iron from its host, $A$. baumannii uses an iron-dependent repressor known as ferric uptake regulator (FUR) and siderophore-mediated iron acquisition systems ${ }^{52}$. The expression of FUR relies on the iron levels in the environment, where more FUR expression occurs when iron levels are ${ }{ }^{5}{ }^{53}$. In the siderophore-mediated iron acquisition system, high-affinity ironchelating compounds are secreted by bacteria and transport iron across cell membranes. Because the binding of siderophores to iron is strong, it can compete with transferrin and lactoferrin and remove iron molecules from carriers $^{51}$. The siderophore-iron complex then 
binds to the corresponding receptors on the bacterial surface and is internalized where the iron is released and used for the internal processes $^{53}$.

Although iron chelators can sequester iron and provide a non-antibiotic alternative treatment approach, a study by Thomson et al. demonstrated that the application of deferoxamine (a widely used iron chelator) is ineffective against A.baumannii; this is believed to be due to the presence of bacterial receptors for deferoxamine ${ }^{54}$. Even though deferoxamine can bind to iron, the deferoxamine-iron complex will bind to the deferoxamine receptors and iron is extracted and used by the bacteria ${ }^{54}$. However, the new generation of iron chelators in combination with other conventional therapeutic approaches may offer therapeutic value in difficult-to-treat MDR cases ${ }^{51}$. Further studies are required to assess the therapeutic value of the newer generation of iron chelators on growth and survival of nosocomial pathogens such as MDR A. baumannii.

\section{Bacteriophage therapy}

Bacteriophages - viral particles that target bacteria - have previously been used as a treatment for bacterial infections ${ }^{55}$. GarciaQuintanilla et al. enumerated several bacteriophages that are effective for specific strains of A. baumannii, including phage AB-1, which is a lytic circular double stranded DNA phage, and phage $\mathrm{AB}-2$ that is lytic to 27 strains of clinical MDR isolates with an adsorption rate of $99 \%$ within 9 min. ${ }^{56}$.

Other reports have described newer phages that are being discovered with a broader host range, chiefly, AP22 that can infect 89 of 130 A. baumannii strains and is considered a potent phage to infect and lyse MDR A. baumannii ${ }^{56}$. Novel phages (e.g. KARL-1) are frequently reposted for use against MDR A. baumannii with increased efficacy when the phage treatment is synergistic with the application of traditional antibiotics ${ }^{55}$.

However, apart from its advantages, phage therapy has its downsides. For instance, not all phages are thought fit to be used for therapeutic purposes as some phages have weak therapeutic potential, thus defeating the primary purpose of phage therapy.
Other phages can convert phagesusceptible bacteria to non-susceptible ones due to superinfection immunity in which genome integration into the host's chromosome takes place, thus blocking the obligate lytic phage to successfully infect and lyse the target bacterium. Phages can also trigger severe immune reaction which may carry major health concerns. However, recent US Food and Drug Administration approval for viral gene therapy treatments, ranging from treatments for blindness to lymphomas, suggests the tremendous potential and future application of viruses as a tool to treat various diseases including difficult cases of MDR infections ${ }^{56}$.

\section{Antimicrobial active herbal compounds against Acinetobacter baumannii}

The high level of acquired and intrinsic carbapenem resistance mechanisms acquired by these bacteria makes their eradication difficult. The pharmaceutical industry has no solution to this problem. Hence, it is an urgent requirement to find a suitable alternative to carbapenem, a commonly prescribed drug for Acinetobacter infection.

L. salicaria shows significant activity against different bacteria but, especially $A$. baumannii and $P$. aeruginosa. Hence its topical form may be used to treat infections of skin and soft tissue (antiseptic), infections of burn wounds, diabetic foot and decubitus wound caused by these MDR bacterial strains. It is already known that this plant has been used as traditional medicine for many indications, but to use it clinically, several in-vitro and invivo test have to be performed ${ }^{57}$.

Saulnier et al. used essential oils of herbs like Syzygium aromaticum, Cinnamomum zeylanicum, and Thymus in nano medicine against multidrug-resistant $A$. baumannii. Cinnamaldehyde prevents the activity of amino acid decarboxylase in the bacteria ${ }^{58}$, but it is unable to disorganize outer membrane of cell or deplete intracellular ATP concentration. Hydroxyl group of carvacrol and eugenol (phenolic compounds) can disrupt the bacterial cell wall. This phenomenon has the potential to decrease intracellular ATP pool and membrane potential $^{59}$. It also results in the leakage of various substances such as ATP, amino acids, ions, and nucleic acids ultimately leading to bacterial death. MIC of active components is 
the same as MIC of those compounds when nano-encapsulated. Lipidic nanocapsules (LNC) can be made more effective by improving their presence in systemic circulation through the modifications on LNC surface. It can be a good alternative of antibiotics $^{60}$.

Curcumin alone had very little antibacterial activity against $A$. baumannii strains with high MIC $(256 \mu \mathrm{g} / \mathrm{ml})$. The antibacterial activity of curcumin is due to several reasons for instance, disruption of folic acid metabolism (shikimate dehydrogenase) pathway and bacterial cell division ${ }^{61}$. Combinatorial use of curcumin and epigallocatechin gallate (EGCG) is very much effective in increasing the inhibition level by many folds, making the MIC $4 \mu \mathrm{g} / \mathrm{ml}$. Synergistic effects to prevent $A$. baumannii growth between curcumin and EGCG without any antagonistic effects ${ }^{62}$. In the same way epicatechin, a tea polyphenol having no antibacterial properties can potentiate theaflavin, increasing its activity against $A$. baumannii and $S$. maltophiliaisolates. The probable mechanism may be that epicatechin inhibits theaflavin oxidation thus enhancing its antibacterial effect, but the exact mechanism of synergy is not yet understood and needs further study ${ }^{63}$.

Khadri, et al. reported the presence of methyl allyl trisulfide (34.61\%) and diallyl disulfide $(31.65 \%)$ with other compounds at relatively lower levels after GC/MS analysis in Allium sativium. It showed significant activity against $P$. aeruginosa in-vitro and can be used for treating infections caused by this pathogen ${ }^{64}$. Aloe vera gel extract were reported to be more active for Gram-positive than Gramnegative bacteria. Ethanol extract was most active followed by methanol extract activity and least inhibition was exhibited by acetone extract $^{65}$. Magnolia dealbata extracts showed good inhibition zone of $>10 \mathrm{~mm}$ against $P$. aeruginosa, Clavibacter michiganensis, $A$. baumannii, A. iwoffii. Generally, the active constituents of $\mathrm{M}$. dealbata like honokiol and magnolol possess selective antimicrobial activities against drug resistant Gram-negative bacterial species and fungal pathogens ${ }^{66}$.

Miyasaki et al. suggested in their studies, norwogonin (5,6,7-trihydroxyflavone) extracted from Scutellaria baicalensis, has an
MIC90 of $128 \mu \mathrm{g} / \mathrm{ml}$ against some strains of $A$. baumannii. Chebulagic acid, chebulinic acid $(65 \%)$ inhibition at $(62.5 \mu \mathrm{g} / \mathrm{ml})$, ellagic acid $(67 \%$ inhibition at $250 \mu \mathrm{g} / \mathrm{ml})$, corilagin, and terchebulin extracted from Terminalia chebula had lower activity against $A$. baumannii invitro. Other constituents of Scutellaria baicalensis, baicalin and baicalein are also found to be active against other bacteria. Corilagin, chebulagic acid, and terchebulin of Terminalia chebula exhibit a two-step killing kinetic. The medical literature reported that many phenolic compounds of plant extracts enhance the potential of synthetic antibiotics against $A$. baumannii in-vitro ${ }^{67}$. For instance, activity of rifampicin, coumermycin, fusidic acid, novobiocin, and chlorobiocin was enhanced by tannic acid and ellagic acid against $A$. baumannii in-vitro ${ }^{68}$. Even synergy was observed between topical mafenide and green tea polyphenol against multi-drug resistance Acinetobacter baumannii in-vitro ${ }^{69}$.

Some Chinese medicines extracted from different plants have been reported to exhibit significant antibacterial activities. The active constituent of Rhizoma coptidis is berberine, an alkaloid possessing various antimicrobial activities. Berberine is also isolated from Berberis fremontii and Hydrastis canadensis ${ }^{70}$. It has anti-Herpes simplex virus effects and at moderate concentrations $(30-45 \mu \mathrm{g} / \mathrm{ml})$ sufficient antibacterial effect was observed along with inhibition of biofilm formation. Plant derived antibacterial molecule are generally weak but work better in synergy with antibiotics $^{70}$. Synergism was also seen for berberine and $\beta$-lactam antibiotics against multi drug resistant $S$. aureus. (+)-Lyoniresinol-3 alpha-O-beta-D-glucopyranoside of Cortex Lycii presented strong antimicrobial effect against multi drug resistant $S$. aureus isolated from patients and some pathogenic fungi, but it did not cause any haemolysis on human RBCs. This also possesses potent antifungal activities against Candida albicans. There is very limited literature concerned with the antibacterial effects of Cortex Moutan. The ethanolic extract of Cortex Moutan suppresses the growth of $S$. aureus. Only paeonol is identified as active ingredients till date, which is responsible for its anti-microbial effects on $C$. albicans, $C$. tropicalis, and C. glabrata etc. ${ }^{71}$. 
A very common medicinal plant A. indica is the source of various active compounds identified by GC/MS analysis possessing versatile effects like anti-bacterial, anti-inflammatory, antioxidant activities. Hence further studies can be performed to see its activity against Gram-negative bacteria and especially A. baumannii ${ }^{72}$.

\section{Prophylactic vaccination}

Vaccination has been one of the most effective approaches in preventing bacterial infections. An essential element in development of an effective vaccine is the choice of the suitable target antigen that is expressed on the bacterial surface. In the mouse model, the first vaccine against $A$. baumannii had a mixture of various bacterial antigens and resulted in a lower tissue bacterial load in vaccinated mice compared to unvaccinated mice ${ }^{73}$.

Since the original trials, other vaccines have been developed with a wide range of targets including the biofilm-associated protein (Bap), outer membrane complexes (OMCs), and the outer membrane vesicles (OMVs). The new generation of vaccines substantially decreases the bacterial loads in vaccinated mice $^{73}$.

Bap protein is an acidic high-molecularweight protein found on the surface of organisms and is considered a key factor in biofilm formation, which is a contributing factor to the emergence of nosocomial infections ${ }^{74}$. Although the presence of Bap is associated with increased virulence, it is still debatable whether the Bap-based vaccine will be effective since some MDR A. baumannii strains do not produce biofilm.

In addition, OMC-targeted vaccines are known to induce immune response that may lead to the proliferation of non-specific natural killer lymphocytes ${ }^{75}$. Alternatively, OMV can increase the antibody titres against $A$. baumannii and minimize the tissue bacterial $\operatorname{loads}^{76}$. Although the results in the mouse model are promising, considering the evidence to date, an ideal vaccine should be a combination of OMV and OMC, as both can combat active bacterial infections. However, OMC-based vaccines exhibit higher endotoxin activity due to the lipopolysaccharide production, and there may be substantial human health and safety concerns ${ }^{77}$.

Although successful in the mouse models, A. baumannii vaccine for human use is still is in its infancy and requires further studies to evaluate its efficacy and safety parameters. This goal may be accomplished with the development of techniques to detoxify the OMC/OMV vaccines to reduce the endotoxin activity as was previously shown for other organisms ${ }^{76}$.

\section{Preventive measures}

A. baumannii is associated with both community- and hospital-acquired infections ${ }^{78}$. In community-acquired cases infections are associated with a high mortality rate, and are often characterized by severe and sudden onset of pneumonia coupled with secondary septicemia $^{79}$. Hospital-acquired infections are frequently associated with monoclonal outbreaks, usually related to an environmental source, or as complex, polyclonal outbreaks, in which epidemic and sporadic clones coexist ${ }^{80}$.

Regardless of the nature of MDR bacterial infections, it is widely accepted that practicing proper hand hygiene is an effective and simple infection control technique. Thom et al. evaluated the presence of $A$. baumannii on the hands and gloves of healthcare workers ${ }^{81}$. They reported on 254 interactions between healthcare workers and 52 patients, in which $30 \%$ of interactions were positive for $A$. baumannii on gloves or hands. More importantly, Haverstick et al. reported that to effectively reduce the rate of nosocomial infections it is necessary for patients to receive hand hygiene supplies and training ${ }^{82}$.

Biswas and Tiwari have reported that $1 \%$ sodium hypochlorite, $2.5 \%$ hydrogen peroxide, and $10 \mathrm{mM}$ chlorine dioxide are effective environmental disinfectants against $A$. baumannii $^{82}$. Leung and Chan have outlined various methods of cleaning hospital air following an outbreak, which include filtration, differential pressure, directional airflow control, and ultraviolet germicidal irradiation ${ }^{83}$. However, they caution against the use of UV radiation because its effectiveness is based on how long the air is exposed to radiation, which is inversely proportional to the air flow rate.

Another control measure for disinfecting hospitals involves hydrogen peroxide vapor. 
Cobrado et al. recently reported successful disinfection of a burn unit contaminated with $A$. baumannii by using an automated aerosolized hydrogen peroxide/silver cation dry-mist system ${ }^{84}$. Improved methods of cleaning by hydrogen peroxide vapour appear to be effective against MDR A. baumannii outbreaks in hospitals when used as an adjunct to standard manual cleaning and disinfection protocols ${ }^{85}$.

Antimicrobial stewardship programmes that restrict excessive antibiotic usage and introduce alternative antibiotic regimens have also been shown to be an effective means of controlling MDR A. baumannii outbreaks ${ }^{86}$. As a long-term strategy, concomitant implementation of strict antimicrobial stewardship in combination with a comprehensive set of intervention measures has been shown to successfully control endemic infections of MDR A. baumannii in a hospital setting within 12 months $^{87}$.

\section{Conclusion}

A. baumannii is an important source of nosocomial infections in hospitals and longterm care facilities. Acquiring multidrug resistance through genetic acquisition / modification leads to increasing number of MDR A. baumannii. Antibiotic therapy against A. baumannii infections is limited, thus alternative treatment options as iron chelation therapy, bacteriophage therapy and antimicrobial active herbal compounds can be useful in some cases. Infection control measures can be costly and challenging.

\section{REFERENCES}

1- K. Sieniawski, K. Kaczka, M. Rucińska, L. Gagis and L. Pomorski, "Acinetobacter baumannii nosocomial infections", Polish Journal of Surgery, 85 (9), 483-490 (2013).

2- S. C. Valentine, D. Contreras, S. Tan, L. J. Real, S. Chu and H. H. Xu, "Phenotypic and molecular characterization of Acinetobacter baumannii clinical isolates from nosocomial outbreaks in Los Angeles County, California", Journal of Clinical Microbiology, 46 (8), 2499-2507 (2008).
3- A. Opazo, M. Domínguez, H. Bello, S. G. Amyes, G. González-Rocha, "OXA-type carbapenemases in Acinetobacter baumannii in South America", The Journal of Infection in Developing Countries, 6 (04), 311-316 (2012).

4- E. Durante-Mangoni and R. Zarrilli, "Global spread of drug-resistant Acinetobacter baumannii: Molecular epidemiology and management of antimicrobial resistance", Future Microbiology, 6 (4), 407-422 (2011).

5- K. M. Hujer, A. M. Hujer, E. A. Hulten, S. Bajaksouzian, J. M. Adams, C. J. Donskey, D. J. Ecker, C. Massire, M. W. Eshoo, R. Sampath and J. M. Thomson, "Analysis of antibiotic resistance genes in multidrug-resistant Acinetobacter $s p$. isolates from military and civilian patients treated at the Walter Reed Army Medical Center", Antimicrobial Agents and Chemotherapy, 50 (12), 4114-4123 (2006).

6- C. F. Prevention, "Antibiotic resistance threats in the United States, 2013", Threat Report, 50-52 (2013).

7- M. E. Falagas, I. A. Bliziotis and I. I. Siempos, "Attributable mortality of Acinetobacter baumannii infections in critically ill patients: A systematic review of matched cohort and case-control studies", Critical Care, 10 (2), R48 (2006).

8- H. Hoffman-Roberts, P. Scoble, Y. P. Tabak, J. Mohr, R. S. Johannes and V. Gupta, "National Prevalence of MultidrugResistant Acinetobacter baumannii Infections in the Ambulatory and Acute Care Settings, Including CarbapenemResistant Acinetobacter Infections, in the United States in 2015", In: Open Forum Infectious Diseases, Oxford University Press, Vol. 3, No. suppl_1, 2016, p. 1488.

9- R. Y. Chinn and L. Sehulster, "Guidelines for Environmental Infection Control in Health-Care Facilities: Recommendations of CDC and Healthcare Infection Control Practices Advisory Committee (HICPAC)", (2003).

10- A. E. Sileem, A. M. Said and M. S. Meleha, "Acinetobacter baumannii in ICU patients: A prospective study highlighting their incidence, antibiotic sensitivity pattern and impact on ICU stay and 
mortality", Egyptian Journal of Chest Diseases and Tuberculosis, 66 (4), 693698 (2017).

11- T. Saied, A. Elkholy, S. F. Hafez, H. Basim, M. O. Wasfy, W. El-Shoubary, A. Samir, G. Pimentel and M. Talaat, "Antimicrobial resistance in pathogens causing nosocomial bloodstream infections in university hospitals in Egypt", American Journal of Infection Control, 39 (9), e61-e65 (2011).

12- M. Talaat, M. El-Shokry, J. El-Kholy, G. Ismail, S. Kotb, S. Hafez, E. Attia and F. C. Lessa, "National surveillance of health care-associated infections in Egypt: Developing a sustainable program in a resource-limited country", ibid., 44 (11), 1296-1301 (2016).

13- A. Y. Peleg, H. Seifert and D. L. Paterson, "Acinetobacter baumannii: Emergence of a successful pathogen", Clinical Microbiology Reviews, 21 (3), 538-582 (2008).

14- M. D. Adams, K. Goglin, N. Molyneaux, K. M. Hujer, H. Lavender, J. J. Jamison, I. J. MacDonald, K. M. Martin, T. Russo, A. A. Campagnari and A. M. Hujer, "Comparative genome sequence analysis of multidrug-resistant Acinetobacter baumannii", Journal of Bacteriology, 190 (24), 8053-8064 (2008).

15- E. S. Snitkin, A. M. Zelazny, C. I. Montero, F. Stock, L. Mijares, P. R. Murray and J. A. Segre, "Genome-wide recombination drives diversification of epidemic strains of Acinetobacter baumannii", Proceedings of the National Academy of Sciences, 108 (33), 1375813763 (2011).

16- P. E. Fournier, D. Vallenet, V. Barbe, S. Audic, H. Ogata, L. Poirel, H. Richet, C. Robert, S. Mangenot, C. Abergel and P. Nordmann, "Comparative genomics of multidrug resistance in Acinetobacter baumannii", PLoS Genet, 2 (1), e7 (2006).

17- K. Aliakbarzade, S. Farajnia, A. K. Nik, F. Zarei and A. Tanomand, "Prevalence of aminoglycoside resistance genes in Acinetobacter baumannii isolates", Jundishapur Journal of Microbiology, 7 (10), e11924 (2014).

18- A. Nemec, L. Dolzani, S. Brisse, P. Van den Broek and L. Dijkshoorn, "Diversity of aminoglycoside-resistance genes and their association with class 1 integrons among strains of pan-European Acinetobacter baumannii clones", Journal of Medical Microbiology, 53 (12), 12331240 (2004).

19- T. Naas and P. Nordmann, "OXA-type Lactamases", Current Pharmaceutical Design, 5, 865-880 (1999).

20- I. Davandeh, B. Erac and S. Ş. AYDEMIR, "Investigation of class-D beta-lactamases causing carbapenem resistance in clinical Acinetobacter baumannii isolates", Turkish Journal of Medical Sciences, 47 (5), 1661-1666 (2017).

21- J. F. Turton, N. Woodford, J. Glover, S. Yarde, M. E. Kaufmann and T. L. Pitt, "Identification of Acinetobacter baumannii by detection of the $b l a_{\mathrm{OXA}^{-}}$ 51-like carbapenemase gene intrinsic to this species", Journal of Clinical Microbiology, 44 (8), 2974-2976 (2006).

22- P. Villalón, S. Valdezate, M. J. MedinaPascual, G. Carrasco, A. Vindel, J. A. Saez-Nieto, "Epidemiology of the Acinetobacter-derived cephalosporinase, carbapenem-hydrolysing oxacillinase and metallo- $\beta$-lactamase genes, and of common insertion sequences, in epidemic clones of Acinetobacter baumannii from Spain", Journal of Antimicrobial Chemotherapy, 68 (3), 550-553 (2013).

23- A. Beceiro, L. Dominguez, A. Ribera, J. Vila, F. Molina, R. Villanueva, J. M. Eiros and G. Bou, "Molecular characterization of the gene encoding a new AmpC $\beta$ lactamase in a clinical strain of Acinetobacter genomic species 3", Antimicrobial Agents and Chemotherapy, 48 (4), 1374-1378 (2004).

24- S. Monem, B. Furmanek-Blaszk, A. Łupkowska, D. Kuczyńska-Wiśnik, K. Stojowska-Swędrzyńska and E. Laskowska, "Mechanisms protecting Acinetobacter baumannii against multiple stresses triggered by the host immune response, antibiotics, and outside host environment", International Journal of Molecular Sciences, 21 (15), 5498 (2020).

25- C. J. Boinett, A. K. Cain, J. Hawkey, N. T. Do Hoang, N. N. Khanh, D. P. Thanh, J. Dordel, J. I. Campbell, N. P. Lan, M. 
Mayho and G. C. Langridge, "Clinical and laboratory-induced colistin-resistance mechanisms in Acinetobacter baumannii", Microbial Genomics, 5 (2), e000246 (2019).

26- J. H. Moffatt, M. Harper, P. Harrison, J. D. Hale, E. Vinogradov, T. Seemann, R. Henry, B. Crane, F. S. Michael, A. D. Cox and B. Adler, "Colistin resistance in Acinetobacter baumannii is mediated by complete loss of lipopolysaccharide production", Antimicrobial Agents and Chemotherapy, 54 (12), 4971-4977 (2010).

27- D. Nie, Y. Hu, Z. Chen, M. Li, Z. Hou, X. Luo, X. Mao and X. Xue, "Outer membrane protein A (OmpA) as a potential therapeutic target for Acinetobacter baumannii infection", Journal of Biomedical Science, 27 (1), 26 (2020).

28- C. F. Xu, S. R. Bilya and W. Xu, "adeABC efflux gene in Acinetobacter baumannii", New Microbes and New Infections, 30, 100549 (2019).

29- A. Nemec, M. Maixnerová, T. J. Van der Reijden, P. J. Van den Broek and L. Dijkshoorn, "Relationship between the AdeABC efflux system gene content, netilmicin susceptibility and multidrug resistance in a genotypically diverse collection of Acinetobacter baumannii strains", Journal of Antimicrobial Chemotherapy, 60 (3), 483-489 (2007).

30- P. G. Higgins, C. Dammhayn, M. Hackel and H. Seifert, "Global spread of carbapenem-resistant Acinetobacter baumannii", ibid., 65 (2), 233-238 (2010).

31- S. E. Cheah, J. Li, B. T. Tsuji, A. Forrest, J. B. Bulitta and R. L. Nation, "Colistin and polymyxin $\mathrm{B}$ dosage regimens against Acinetobacter baumannii: Differences in activity and the emergence of resistance", Antimicrobial Agents and Chemotherapy, 60 (7), 3921-3933 (2016).

32- W. Ni, Y. Han, J. Zhao, C. Wei, J. Cui, R. Wang and Y. Liu, "Tigecycline treatment experience against multidrug-resistant Acinetobacter baumannii infections: A systematic review and meta-analysis", International Journal of Antimicrobial Agents, 47 (2), 107-116 (2016).
33- W. F. Penwell, A. B. Shapiro, R. A. Giacobbe, R. F. Gu, N. Gao, J. Thresher, R. E. McLaughlin, M. D. Huband, B. L. DeJonge, D. E. Ehmann and A. A. Miller, "Molecular mechanisms of sulbactam antibacterial activity and resistance determinants in Acinetobacter baumannii", Antimicrobial Agents and Chemotherapy, 59 (3), 1680-1689 (2015).

34- K. Kengkla, K. Kongpakwattana, S. Saokaew, A. Apisarnthanarak and N. Chaiyakunapruk, "Comparative efficacy and safety of treatment options for MDR and XDR Acinetobacter baumannii infections: A systematic review and network meta-analysis", Journal of Antimicrobial Chemotherapy, 73 (1), 2232 (2018).

35- F. Perez, A. M. Hujer, K. M. Hujer, B. K. Decker, P. N. Rather and R. A. Bonomo, "Global challenge of multidrug-resistant Acinetobacter baumannii", Antimicrobial Agents and Chemotherapy, 51 (10), 34713484 (2007).

36- R. L. Nation, T. Velkov and J. Li, "Colistin and polymyxin B: Peas in a pod, or chalk and cheese?, Clinical Infectious Diseases, 59 (1), 88-94 (2014).

37- M. D. Adams, G. C. Nickel, S. Bajaksouzian, H. Lavender, A. R. Murthy, M. R. Jacobs and R. A. Bonomo, "Resistance to colistin in Acinetobacter baumannii associated with mutations in the PmrAB two-component system", Antimicrobial Agents and Chemotherapy, 53 (9), 3628-3634 (2009).

38- A. O. Javan, S. Shokouhi and Z. Sahraei, "A review on colistin nephrotoxicity", European Journal of Clinical Pharmacology, 71 (7), 801-810 (2015).

39- A. Zorgani, A. Abofayed, A. Glia, A. Albarbar and S. Hanish, "Prevalence of device-associated nosocomial infections caused by gram-negative bacteria in a trauma intensive care unit in Libya", Oman Medical Journal, 30 (4), 270 (2015).

40- T. C. Menegucci, J. Albiero, L. B. Migliorini, J. L. Alves, G. F. Viana, J. Mazucheli, F. E. Carrara-Marroni, C. L. Cardoso and M. C. Tognim, "Strategies for the treatment of polymyxin B-resistant Acinetobacter baumannii infections", 
International Journal of Antimicrobial Agents, 47 (5), 380-385 (2016).

41- N. M. Attia and A. ElBaradei, "Fluoroquinolone resistance conferred by gyrA, parC mutations, and AbaQ efflux pump among Acinetobacter baumannii clinical isolates causing ventilatorassociated pneumonia", Acta Microbiologica et Immunologica Hungarica, 2019, 1-5 (2019).

42- S. B. Josheghani, R. Moniri, F. Firoozeh, M. Sehat, K. Dastehgoli, H. Koosha and R. K. Farahani, "Emergence of bla $\mathrm{OXA}^{-}$ carrying carbapenem resistance in multidrug - resistant Acinetobacter baumannii in the intensive care unit", Iranian Red Crescent Medical Journal, 19 (5), e27327 (2017).

43- G. C. Leite, M. S. Oliveira, L. V. Perdigão-Neto, C. K. Rocha, T. Guimarães, C. Rizek, A. S. Levin and S. F. Costa, "Antimicrobial combinations against pan-resistant Acinetobacter baumannii isolates with different resistance mechanisms", PLoS ONE, 11 (3), e0151270 (2016).

44- S. Abdellatif, A. Trifi, F. Daly, K. Mahjoub, R. Nasri and S. B. Lakhal, "Efficacy and toxicity of aerosolised colistin in ventilator-associated pneumonia: A prospective, randomised trial", Annals of Intensive Care, 6 (1), 1-1 (2016).

45- T. Demirdal, U. S. Sari and S. A. Nemli, "Is inhaled colistin beneficial in ventilator associated pneumonia or nosocomial pneumonia caused by Acinetobacter baumannii?", Annals of Clinical Microbiology and Antimicrobials, 15 (1), 11 (2016).

46- W. Y. Kim, J. Y. Moon, J. W. Huh, S. H. Choi, C. M. Lim, Y. Koh, Y. P. Chong and S. B. Hong, "Comparable efficacy of tigecycline versus colistin therapy for multidrug-resistant and extensively drugresistant Acinetobacter baumannii pneumonia in critically ill patients", PLoS ONE, 11 (3), e0150642 (2016).

47- R. Aljindan, H. Bukharie, A. Alomar and B. Abdalhamid, "Prevalence of digestive tract colonization of carbapenem-resistant Acinetobacter baumannii in hospitals in
Saudi Arabia", Journal of Medical Microbiology, 64 (4), 400-406 (2015).

48- K. M. Krause, A. W. Serio, T. R. Kane and L. E. Connolly, "Aminoglycosides: An overview", Cold Spring Harbor Perspectives in Medicine, 6 (6), a027029 (2016).

49- M. S. Ramirez and M. E. Tolmasky, "Aminoglycoside modifying enzymes", Drug Resistance Updates, 13 (6), 151-171 (2010).

50- G. P. Neupane and D. M. Kim, "In-vitro time-kill activities of ciprofloxacin alone and in combination with the iron chelator deferasirox against Vibrio vulnificus", European Journal of Clinical Microbiology \& Infectious Diseases, 29 (4), 407-410 (2010).

51- M. García-Quintanilla, M. R. Pulido, R. López-Rojas, J. Pachón and M. J. McConnell, "Emerging therapies for multidrug resistant Acinetobacter baumannii", Trends in Microbiology, 21 (3), 157-163 (2013).

52- E. P. Skaar, "The battle for iron between bacterial pathogens and their vertebrate hosts", PLoS Pathog., 6 (8), e1000949 (2010).

53- C. C. Nwugo, J. A. Gaddy, D. L. Zimbler and L. A. Actis, "Deciphering the iron response in Acinetobacter baumannii: A proteomics approach", Journal of Proteomics, 74 (1), 44-58 (2011).

54- M. G. Thompson, B. W. Corey, Y. Si, D. W. Craft and D. V. Zurawski, "Antibacterial activities of iron chelators against common nosocomial pathogens", Antimicrobial Agents and Chemotherapy, 56 (10), 5419-5421 (2012).

55- M. Jansen, A. Wahida, S. Latz, A. Krüttgen, H. Häfner, E. M. Buhl, K. Ritter and H. P. Horz, "Enhanced antibacterial effect of the novel T4-like bacteriophage KARL-1 in combination with antibiotics against multi-drug resistant Acinetobacter baumannii", Scientific Reports, 8 (1), 1-2 (2018).

56- F. Peng, Z. Mi, Y. Huang, X. Yuan, W. Niu, Y. Wang, Y. Hua, H. Fan, C. Bai and Y. Tong, "Characterization, sequencing and comparative genomic analysis of vB_AbaM-IME-AB2, a novel lytic 
bacteriophage that infects multidrugresistant Acinetobacter baumannii clinical isolates", BMC Microbiology, 14 (1), 181 (2014).

57- E, Guclu, H. Genc, M. Zengin and O. Karabay, "Antibacterial activity of Lythrum salicaria against multidrugresistant Acinetobacter baumannii and Pseudomonas aeruginosa", Annual Research \& Review in Biology, 4 (7), 1099-1105 (2014).

58- S. Burt, "Essential oils: Their antibacterial properties and potential applications in foods - A review", International Journal of Food Microbiology, 94 (3), 223-253 (2004).

59- A. O. Gill and R. A. Holley, "Disruption of Escherichia coli, Listeria monocytogenes and Lactobacillus sakei cellular membranes by plant oil aromatics", ibid., 108 (1), 1-9 (2006).

60 A. Montagu, P. Saulnier, V. Cassisa, E. Rossines, M. Eveillard and M. L. JolyGuillou, "Aromatic and terpenic compounds loaded in lipidic nanocapsules: Activity against multi-drug resistant Acinetobacter baumannii assessed in-vitro and in a Murine model of sepsis", Journal of Nanomedicine \& Nanotechnology, 5 (3), 206 (2014).

61- R. De, P. Kundu, S. Swarnakar, T. Ramamurthy, A. Chowdhury, G. B. Nair and A. K. Mukhopadhyay, "Antimicrobial activity of curcumin against Helicobacter pylori isolates from India and during infections in mice", Antimicrobial Agents and Chemotherapy, 53 (4), 1592-1597 (2009).

62- J. W. Betts and D. W. Wareham, "In-vitro activity of curcumin in combination with epigallocatechin gallate (EGCG) versus multidrug-resistant Acinetobacter baumannii", BMC Microbiology, 14 (1), 172 (2014).

63- J. W. Betts, S. M. Kelly and S. J. Haswell, "Antibacterial effects of theaflavin and synergy with epicatechin against clinical isolates of Acinetobacter baumannii and Stenotrophomonas maltophilia",

International Journal of Antimicrobial Agents, 38 (5), 421-425 (2011).

64- S. Khadri, N. Boutefnouchet and M. Dekhil, "Antibacterial activity evaluation of alliumsativum essential oil compared to different Pseudomonas aeruginosa strains in eastern Algeria", Scientific Study \& Research: Chemistry \& Chemical Engineering, Biotechnology, Food Industry, 11 (4), 421-428 (2010).

65- R. Lawrence, P. Tripathi and E. Jeyakumar, "Isolation, purification and evaluation of antibacterial agents from Aloe vera", Brazilian Journal of Microbiology, 40 (4), 906-915 (2009).

66- M. D. Jacobo-Salcedo, L. A. GonzalezEspindola, A. J. Alonso-Castro, M. D. Gonzalez-Martinez, F. Domínguez and A. Garcia-Carranca, "Antimicrobial activity and cytotoxic effects of Magnolia dealbata and its active compounds", Natural Product Communications, 6 (8), 1934578X1100600818 (2011).

67- Y. Miyasaki, J. D. Rabenstein, J. Rhea, M. L. Crouch, U. M. Mocek, P. E. Kittell, M. A. Morgan, W. S. Nichols, M. M. Van Benschoten, W. D. Hardy and G. Y. Liu, "Isolation and characterization of antimicrobial compounds in plant extracts against multidrug-resistant Acinetobacter baumannii", PLoS ONE, 8 (4), e61594 (2013).

68- S. Chusri, I. Villanueva, S. P. Voravuthikunchai and J. Davies, "Enhancing antibiotic activity: A strategy to control Acinetobacter infections", Journal of Antimicrobial Chemotherapy, 64 (6), 1203-1211 (2009).

70- K. Lewis and F. M. Ausubel, "Prospects for plant-derived antibacterials", Nature Biotechnology, 24 (12), 1504-1507 (2006).

71- P. C. Chan, L. M. Huang, H. C. Lin, L. Y. Chang, M. L. Chen, C. Y. Lu, P. I. Lee, J. M. Chen, C. Y. Lee, H. J. Pan and J. T. Wang, "Control of an outbreak of pandrug-resistant Acinetobacter baumannii colonization and infection in a neonatal intensive care unit", Infection Control \& Hospital Epidemiology, 28 (4), 423-429 (2007).

72- P. Nand, S. Drabu and R. K. Gupta, "Insignificant anti-acne activity of Azadirachta indica leaves and bark", Journal of Pharmaceutical Negative Results, 3 (1), 29-33 (2012). 
73- T. A. Ahmad, D. M. Tawfik, S. A. Sheweita, M. Haroun and L. H. El-Sayed, "Development of immunization trials against Acinetobacter baumannii", Trials in Vaccinology, 5, 53-60 (2016).

74- K. A. Brossard and A. A. Campagnari, "The Acinetobacter baumannii biofilmassociated protein plays a role in adherence to human epithelial cells", Infection and Immunity, 80 (1), 228-233 (2012).

75- M. J. McConnell, C. Rumbo, G. Bou and J. Pachón, "Outer membrane vesicles as an acellular vaccine against Acinetobacter baumannii", Vaccine, 29 (34), 5705-5710 (2011).

76- M. J. McConnell, L. Actis and J. Pachón, "Acinetobacter baumannii: Human infections, factors contributing to pathogenesis and animal models", FEMS Microbiology Reviews, 37 (2), 130-155 (2013).

77- M. R. Pulido, M. García-Quintanilla, J. Pachón and M. J. McConnell, "A lipopolysaccharide-free outer membrane vesicle vaccine protects against Acinetobacter baumannii infection", Vaccine, 38 (4), 719-724 (2020).

78- A. Howard, M. O'Donoghue, A. Feeney and R. D. Sleator, "Acinetobacter baumannii: An emerging opportunistic pathogen", Virulence, 3 (3), 243-250 (2012).

79- N. M. Anstey, B. J. Currie, M. Hassell, D. Palmer, B. Dwyer and H. Seifert, "Community-acquired bacteremic Acinetobacter pneumonia in tropical Australia is caused by diverse strains of Acinetobacter baumannii, with carriage in the throat in at-risk groups", Journal of Clinical Microbiology, 40 (2), 685-686 (2002).

80- E. Tacconelli, M. A. Cataldo, S. J. Dancer, G. De Angelis, M. Falcone, U. Frank, G. Kahlmeter, A. Pan, N. Petrosillo, J. Rodríguez-Baño and N. Singh, "ESCMID guidelines for the management of the infection control measures to reduce transmission of multidrug-resistant Gramnegative bacteria in hospitalized patients",
Clinical Microbiology and Infection, 20 (1), 1-55 (2014).

81- K. A. Thom, C. Rock, S. S. Jackson, J. K. Johnson, A. Srinivasan, L. Magder, M. C. Roghmann, R. A. Bonomo and A. D. Harris, "Factors leading to transmission risk of Acinetobacter baumannii", Critical Care Medicine, 45 (7), e633 (2017).

82- S. Haverstick, C. Goodrich, R. Freeman, S. James, R. Kullar and M. Ahrens, "Patients' hand washing and reducing hospital-acquired infection", Critical Care Nurse, 37 (3), e1-e8 (2017).

83- M. Leung and A. H. J. M. Chan, "Control and management of hospital indoor air quality", Medical Science Monitor, 12 (3), SR17-SR23 (2006).

84- L. Cobrado, A. Pinto Silva, C. Pina-Vaz and A. Rodrigues, "Effective disinfection of a burn unit after two cases of sepsis caused by multi-drug-resistant Acinetobacter baumannii", Surgical Infections, 19 (5), 541-543 (2018).

85- R. Tekin, T. Dal, F. Bozkurt, Ö. Deveci, Y. Palanc1, E. Arslan, C. T. Selçuk and S. Hoşoğlu, "Risk factors for nosocomial burn wound infection caused by multidrug resistant Acinetobacter baumannii", Journal of Burn Care \& Research, 35 (1), e73-e80 (2014).

86- A. Ogutlu, E. Guclu, O. Karabay, A. C. Utku, N. Tuna and M. Yahyaoglu, "Effects of Carbapenem consumption on the prevalence of Acinetobacter infection in intensive care unit patients", Annals of Clinical Microbiology and Antimicrobials, 13 (1), 1-6 (2014).

87- S. Cheon, M. J. Kim, S. J. Yun, J. Y. Moon and Y. S. Kim, "Controlling endemic multidrug-resistant Acinetobacter baumannii in intensive care units using antimicrobial stewardship and infection control", The Korean Journal of Internal Medicine, 31 (2), 367 (2016)w. 


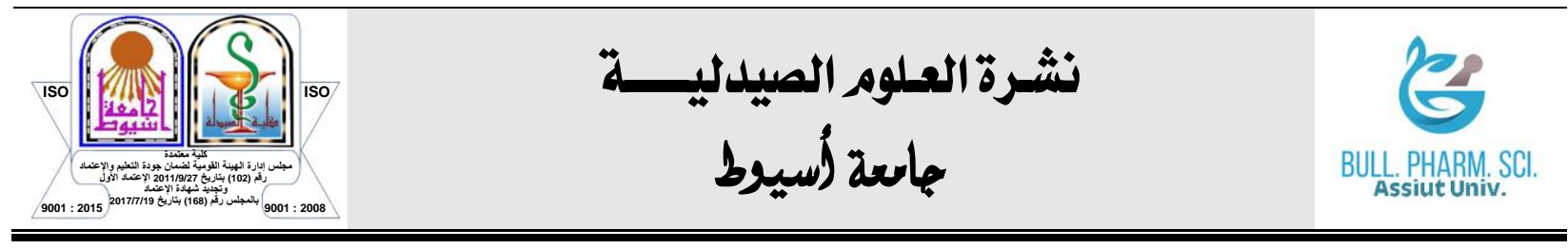

مقاومة الاسينيتوباكتر بومناي للمضادات الحيوية:

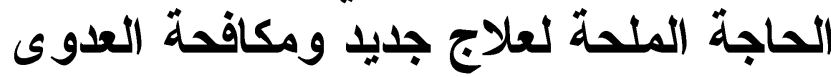

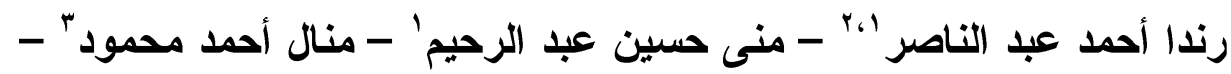

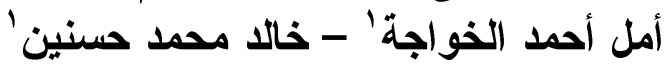

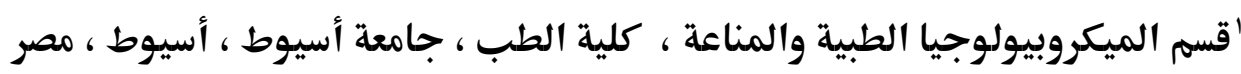

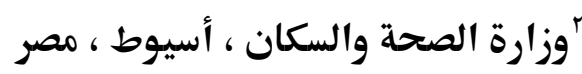

"قسم أمراض الصدر ، كلية الطب ، جامعة أسيوط ، أسيوط ، مصر ، أسرو

أصبحت عدوى الاسينيتو باكتر بومناي مصدر قلق صحي ناشئ في المستثفيات في جميع أنحاء

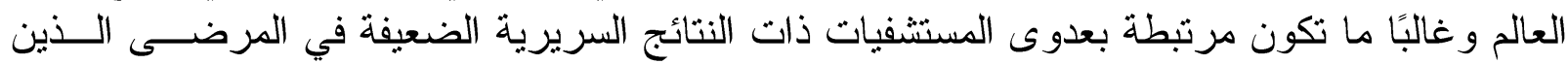

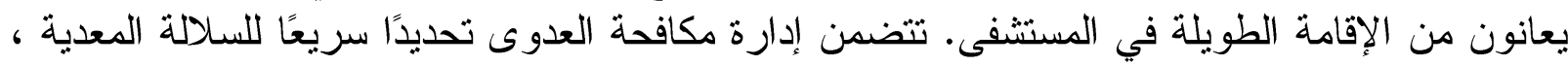

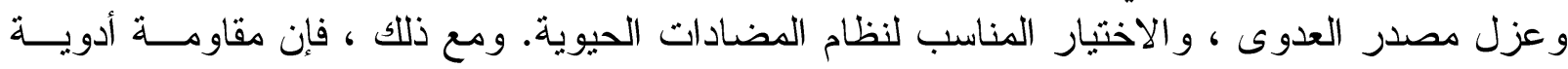

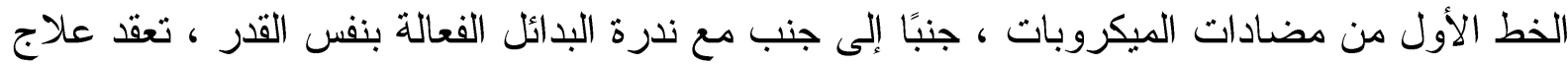

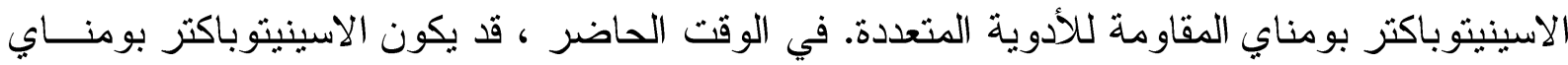

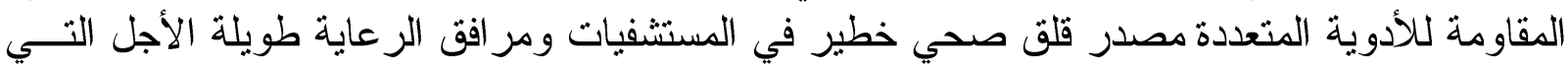

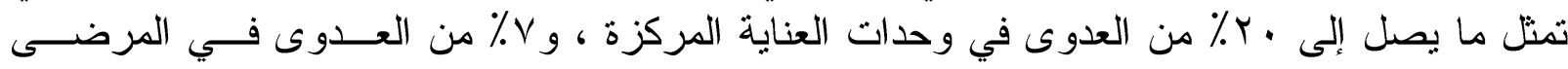

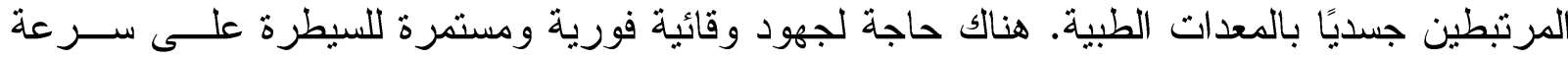

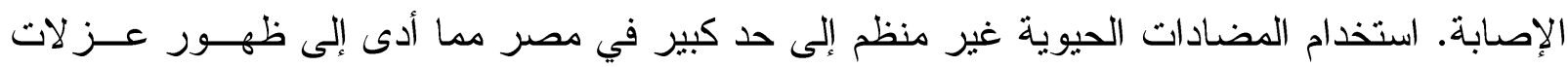

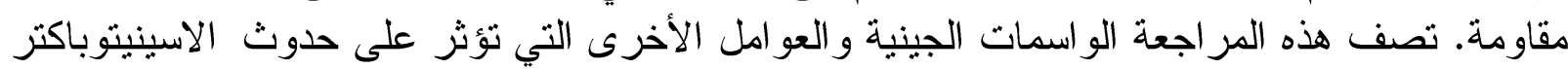

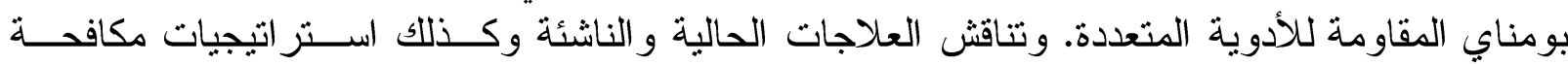
العدوى. 